\title{
La meditación en espacios educativos como factor protector ante la violencia
}

\author{
Meditation in Educational Places as a Protective Factor Against Violence
}

\section{Meditação nos espaços educacionais como fator de proteção contra a violência}

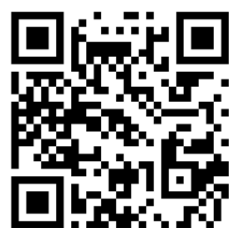

\author{
Francesca Randazzo-Eisemann \\ Universidad Nacional Autónoma de Honduras \\ Tegucigalpa, Honduras \\ elfriede.randazzo@unah.edu.hn \\ https://orcid.org/0000-0002-9572-0507
}

Recibido • Received • Recebido: 19 / 11 / 2019

Corregido • Revised $\cdot$ Revisado: 10 / 07 / 2021

Aceptado • Accepted • Aprovado: 12 / $08 / 2021$

\begin{abstract}
Resumen:
Introducción. En este artículo se explora el impacto que tiene la meditación en el ámbito educativo como factor protector ante la violencia. Se trata de indagar cómo la meditación promueve el desarrollo de la cultura de paz en el centro educativo, así como evidenciar su correlación positiva en la calidad de la educación. Se busca dilucidar si al meditar se pueden favorecer ambientes escolares de convivencia y resolución pacífica de los conflictos, para así contribuir a mejorar el rendimiento y los logros de la población estudiantil, así como también a fomentar un mayor bienestar para el resto de la comunidad educativa. Metodología. Se trata de un estudio exploratorio de carácter cuali-cuantitativo y construccionista, basado en entrevistas y cuestionarios aplicados a agentes parte del proceso social: estudiantado, profesorado y voluntariado. Resultados. Se concluye, según la percepción de estos sujetos, que la meditación en contextos educativos promueve factores protectores ante la violencia, tales como paciencia, sensibilidad, equilibrio emocional, bienestar y relajación. Al haber calma y armonía, bajan los niveles de estrés, agresividad, ansiedad e impulsividad. Promoviendo la convivencia pacífica, las personas son menos conflictivas, más agradables, comprensivas, respetuosas, sociables. Se mejora el estado de ánimo y la autoestima, lo que permite una mejor interacción social: más y mejores relaciones entre pares, aceptación, respeto, colaboración, apoyo, solidaridad e integración.
\end{abstract}

Palabras claves: Convivencia pacífica; meditación; estrés; resiliencia; enseñanza. 
http://doi.org/10.15359/ree.25-3.24

http://www.una.ac.cr/educare

educare@una.ac.cr

\begin{abstract}
:
Introduction. This article explores the impact that meditation has on education as a protective factor against violence. The aim is to determine how meditation promotes the development of peace culture in educational centers, and to demonstrate its positive correlation in the education quality. The objective is to clarify whether meditating can favor school environments of peaceful coexistence and conflict resolution contributing to improve the performance and achievements of the school attendants, as well as promoting greater well-being for the rest of the educational community members. Methods. This is an exploratory qualitative-quantitative constructionist study, based on interviews and questionnaires applied to agents involved in the social process: students, teachers, and volunteers facilitating the meditation technique. Results. According to their perception, it is concluded that meditation in educational contexts promotes protective factors against violence, such as patience, sensitivity, emotional balance, well-being, and relaxation. By having calm and harmony, the levels of stress, aggressiveness, anxiety, and impulsivity decrease. It makes people less conflictive, more pleasant, understanding, respectful, sociable, promoting peaceful coexistence. It improves mood and self-esteem. This allows better social interaction: more and better peer relationships, acceptance, respect, collaboration, support, solidarity, and integration.
\end{abstract}

Keywords: Peaceful coexistence; meditation; stress; resilience; teaching.

\begin{abstract}
Resumo:
Introdução. Este artigo explora o impacto que a meditação tem na educação como um fator protetor contra a violência. Trata-se de investigar como a meditação promove o desenvolvimento de uma cultura de paz no centro educacional e demonstrar sua correlação positiva na qualidade da educação. Procura-se elucidar como, através da meditação, os ambientes escolares de convivência e resolução pacífica de conflitos contribuem para melhorar o desempenho; permitindo favorecer as realizações da população estudantil, bem como promover maior bem-estar para o restante dos membros da comunidade educacional. Metodologia. Este estudo exploratório é qualitativo-quantitativo e construcionista, tem base em entrevistas e questionários aplicados a agentes que fazem parte do processo social: estudantes, professores e voluntários. Resultados. Conclui-se, segundo a percepção desse sujeitos, que a meditação em contextos educacionais promove fatores de proteção contra a violência, como paciência, sensibilidade, equilíbrio emocional, bem-estar e relaxamento. Por ter calma e harmonia, os níveis de estresse, agressividade, ansiedade e impulsividade diminuem. Promovendo a coexistência pacífica, as pessoas são menos conflituosas, mais agradáveis, compreensivas, respeitosas e sociáveis. Melhorar o humor e a auto-estima permite uma melhor interação social: mais e melhores relacionamentos entre colegas, aceitação, respeito, colaboração, apoio, solidariedade e integração.
\end{abstract}

Palavras-chave: Convivência pacífica; meditação; estresse; resiliência; ensino.

\title{
Violencia: factores de riesgo, prevención y resiliencia
}

La violencia entre jóvenes es alarmante. Así lo afirman y sustentan los estudios de Organización Mundial de la Salud (OMS), según la cual cada año se cometen en todo el mundo 200000 homicidios entre jóvenes de 10 a 29 años (OMS, 2017). El homicidio es la cuarta causa de muerte en este grupo etario, y el $83 \%$ de estas víctimas son del sexo masculino. Por cada joven 
que se asesina, otra gran cantidad sufre lesiones que requieren tratamiento hospitalario, pero también ocurren otras formas de violencia cuyas marcas son menos evidentes. Con respecto al género, "entre un $3 \%$ y un $24 \%$ de las mujeres declaran que su primera experiencia sexual fue forzada" (OMS, 2020, párr. 5). En Latinoamérica, y de manera destacada las edades comprendidas entre los 15 y los 24 años, constituyen la franja de población más expuesta a la violencia.

La violencia en los entornos educativos es un fenómeno global (Kibriya et al., 2011). Los centros educativos pueden ser lugares de riesgo, donde se experimenta acoso sexual, venta de drogas y robos, entre otros. Incluso, en algunos países, bandas criminales realizan labores de reclutamiento, vigilancia e inteligencia, tráfico de drogas y extorsión (Consejo Noruego para Refugiados [NRC], 2016). Otras formas de violencia son las burlas, amenazas, intimidaciones, riñas, apodos, insultos, agresiones físicas, psicológicas y sexuales (Ayestas, 2007). Es importante destacar que las investigaciones de Benbenishty y Astor (2005) muestran que las formas más extremas de violencia están asociadas al entorno social inmediato de las escuelas, mientras que las formas de violencia leve y moderada se asocian con el clima institucional.

Se habla del continuum de la violencia: violencia en la infancia debida al acoso escolar (bullying), violencia dentro de la familia o violencia doméstica, violencia dentro de la comunidad, violencia cometida por las bandas juveniles, violencia del crimen organizado, y violencia entre naciones (Abt y Winship, 2016). De allí la necesidad de un enfoque que permita abordar los distintos sistemas. Para atacar las raíces de la violencia, la OMS ha propuesto el uso del modelo ecológico (Bronfenbrenner, 1979) para intentar comprender su naturaleza polifacética (OMS, 2002), planteando además que existen factores de riesgo, protectores y de resiliencia. Con relación a la teoría, los estudios indican efectivamente que los factores protectores inhiben a los factores de riesgo (Gaxiola Romero y Frías Armenta, 2008), mientras que los de resiliencia permiten la capacidad de sobreponerse a resultados adversos. Por "resiliencia" en específico se entiende la capacidad de afrontar la adversidad, sobreponerse a momentos críticos, adaptarse luego de experimentar alguna situación adversa o enfrentarse a un agente perturbador, reflejándose en un mayor equilibrio emocional.

Ayestas (2007) considera que los factores de riesgo que inciden en la ocurrencia de conductas violentas en los centros educativos son personales, familiares, del centro educativo y de la comunidad, reflejando el uso del modelo ecológico. Efectivamente, los centros educativos se encuentran en partes medulares del sistema, concretamente en los niveles de las relaciones y de la comunidad. La enseñanza formal es ciertamente un ámbito privilegiado dentro de los espacios cotidianos de las interacciones que un menor tiene con su entorno inmediato (microsistema), y los lugares legitimados para proporcionar la conexión con las estructuras sociales (mesosistema) (Bronfenbrenner, 1979). 
http://doi.org/10.15359/ree.25-3.24

http://www.una.ac.cr/educare

educare@una.ac.cr

Para la OMS (2017) un factor de riesgo es cualquier rasgo, característica o exposición de un individuo que aumente su probabilidad de sufrir una enfermedad o lesión. Justamente en el espacio educativo se detectan factores de riesgo, entendidos como características o cualidades unidas a una elevada probabilidad de dañar la salud o la integridad. Entre los factores de riesgo para sufrir maltrato en este entorno se encuentran, al menos, los siguientes: fracaso escolar, amigos en situación de riesgo de llevar a cabo conductas antisociales o autodestructivas, aislamiento social, dificultades para las relaciones sociales, y tener menos de seis años (Abt y Winship, 2016).

En el estudio del Banco Mundial (2011) se determina que, entre los factores de riesgo a nivel comunitario que pueden hacer a un joven más susceptible de ser víctima de violencia o hacerse miembro de una banda, se encuentran los bajos índices de inscripción y conclusión de la escuela secundaria, por un lado, y la violencia en las escuelas, por otro. La violencia juvenil se correlaciona con niveles más bajos de educación y la ausencia de influencias sociales positivas por parte de docentes y pares. Asimismo, el autor señala que, si bien los centros educativos pueden estar sumidos en la violencia, también representan un importante factor de protección en las vidas de la juventud en riesgo: los estudios muestran que este es uno de los espacios con mayor impacto para reducir la violencia.

Justamente, dentro del ámbito educativo se pueden promover los factores protectores, entendidos como aquellas características capaces de revertir circunstancias potencialmente negativas y transformarlas; es decir que, a pesar de los riesgos, se constituyen en influencias que modifican, mejoran o alteran la respuesta de una persona a algún peligro. Entre los factores protectores se destacan las experiencias escolares positivas y las buenas relaciones con iguales, la importante implicación en el juego, y los buenos resultados en los estudios (Gaxiola Romero y Frías Armenta, 2008).

Finalmente, como factores de resiliencia, es decir, de desarrollar la capacidad de afrontar este tipo situaciones de riesgo y continuar proyectándose en el futuro a pesar de las adversidades, en los entornos escolares sobresale la existencia de personas adultas significativas y la disposición de herramientas psicoemocionales (Abt y Winship, 2016).

\section{Prevención y resiliencia: Las meditaciones en el entorno educativo}

Las personas no son violentas per se, sino que "se encuentran en situaciones" violentas. Por lo tanto, no se trata de un problema del ser, sino del estar, lo cual cambia el foco del problema hacia la convivencia y la forma de prevenir las situaciones en que se genera la violencia. Esto trasciende el sistema escolar e implica la participación de todos los actores de la sociedad, si bien el espacio de la educación formal es fundamental y privilegiado, pues permite brindar herramientas psicoemocionales y psicosociales. 
Un aspecto que determina la reducción de violencia en los centros educativos reside en brindar espacios de aprendizaje integral, así como informar, enseñar en la niñez a controlarse, a través de la oportunidad para ejercitar el autocontrol en situaciones de alto riesgo, con base en habilidades, reforzando la seguridad y fortaleciendo la resiliencia mediante el cambio de las normas sociales y de apoyo social (Organización Panamericana de la Salud [OPS], 2016). Por ello, proporcionar instrumentos para promover la resiliencia parece ser una de las respuestas.

Con el fin de mejorar el buen clima escolar son fundamentales las actividades prosociales, en los centros educativos y fuera de ellos, con el objetivo de fortalecer la ayuda mutua, creando vínculos con la comunidad. Contar con un abanico de acciones positivas dentro del repertorio de las experiencias personales es por sí solo capaz de elevar la autoestima. De allí el interés de estudiar las meditaciones promovidas por la organización sin fines de lucro Manos sin Fronteras Internacional, como parte de su trabajo de promoción social en centros educativos alrededor del mundo (Manos sin Fronteras, 2020).

Los estudios de Almeida y Stadnik (2017), Ferreira (2016), Nichel et al. (2013), Nichel y Stadnik $(2014,2016)$ y Rocha (2014) y describen los beneficios de estas meditaciones en contextos escolares. Las autoras han realizado detallados trabajos de monitoreo y sistematización de las experiencias conducidas por Manos sin Fronteras Internacional en la educación formal infantil, en varias etapas del desarrollo sociocognitivo, iniciando con grupos de niñas y niños en edad preescolar, y continuando en todos los espacios de la formación educativa sucesiva.

Los estudios científicos indican que la práctica de la meditación a corto plazo disminuye considerablemente el estrés, la ansiedad, la tendencia a padecer accesos de cólera y los riesgos de recaída; además fortalece el sistema inmunitario, mejora la tensión arterial en los sujetos hipertensos, promueve las emociones positivas y la capacidad de atención (Ricard, 2009).

En palabras de la fundadora de Manos sin Fronteras:

La meditación reduce el estrés y, otra cosa también, para muchas madres y especialmente las mujeres mejora el sueño; y esto ha estado demostrado que tiene un efecto muy positivo sobre nuestro cerebro. Es lo que más gasta en calorías - nuestro cerebro-y también es el que gasta más azúcar. En muchas universidades lo han estudiado, como la de Harvard: meditar activa varias zonas de nuestro cerebro que, a la larga, repercuten de una manera directa en nuestra salud y nuestra forma de ser, sobre todo cuando tenemos ansiedad. (La Jardinera, 2020, “QQuién soy yo?”, párr. 4).

Efectivamente, aunque la primera finalidad de la meditación sea la de transformar nuestra experiencia del mundo, lo cierto es que la experiencia meditativa también ejerce efectos beneficiosos sobre la salud. Desde hace más o menos unos diez años, importantes universidades americanas, como la Universidad de Madison en Wisconsin, y las de Princeton, Harvard y Berkeley, 
http://doi.org/10.15359/ree.25-3.24

http://www.una.ac.cr/educare

educare@una.ac.cr

al igual que algunos centros de Zúrich y Maastricht, en Europa, están investigando mucho sobre la meditación, así como sobre su acción a corto y largo plazo en el cerebro (Ricard, 2009, p. 13).

A través del trabajo de decenas de voluntarias y voluntarios, en instituciones educativas anuentes a explorar esta experiencia a nivel mundial, Manos sin Fronteras ha promovido durante la última década la meditación a través de varias actividades y técnicas de aplicación sencilla; entre ellas la forma básica denominada 3-5-3 (3 respiraciones, 5 minutos de descanso mental, 3 respiraciones), y la aplicación para el celular 5 minutos, yo medito (Eumedito, 2020), que incluye la meditación básica de 5 minutos, la posibilidad de incrementar el tiempo de meditación, la meditación guiada de La Jardinera (2020), explicaciones de la técnica y un meditómetro.

\section{Metodología}

La metodología de este estudio exploratorio es de carácter cuali-cuantitativo y construccionista, basada en entrevistas semiestructuradas y cuestionarios con preguntas cerradas y abiertas, aplicados a agentes sociales parte del proceso estudiado: docentes, estudiantes y personas voluntarias facilitadoras de la técnica de meditación. Su objetivo es determinar si la meditación en el ámbito educativo formal fomenta los factores protectores y de resiliencia ante la violencia. Para ello, a través de un estudio de percepción de los propios actores, se retoman las principales experiencias de las meditaciones realizadas por Manos sin Fronteras en escuelas, colegios y universidades, analizando la recepción de la meditación en los centros educativos y su impacto, especialmente como factor protector ante la violencia.

Gracias al Instituto de Estimulación Neural y Terapias Naturales se pudieron contactar las oficinas nacionales de Manos sin Fronteras, las cuales se encargaron de establecer los lazos entre la investigadora y las personas participantes vía electrónica para la aplicación de instrumentos. Tanto la convocatoria como la aplicación se realizó por correo electrónico, WhatsApp y formularios virtuales.

Si bien la meditación ha sido implementada en los últimos años en diferentes contextos educativos del mundo por Manos sin Fronteras, en este trabajo se retoman exclusivamente las experiencias de Brasil (São Paulo, Curitiba, Paraná, Piraquara, Contenda, Lagoa da Prata, Uberaba, Minas Gerais), Colombia (Medellín, Bogotá, Roldanillo, Neiva, Cali, Dosquebradas, Risaralda), Italia (Podenzano, Piacenza), México (Cancún, Quintana Roo; Guadalajara, Jalisco), Honduras (Tegucigalpa) y Guatemala (Guatemala).

El diseño es participativo e incluyente, pues se discutieron los instrumentos y marcos teóricos (estado del arte y marco analítico) en conjunto con el Instituto de Estimulación Neural y Terapias Naturales, así como con algunas personas voluntarias de Manos sin Fronteras en diversos países. Los instrumentos fueron pasados por medios electrónicos, permitiendo la recopilación de información que de otra manera era inviable, por razones geográficas.

6 
Con el fin de escuchar y tomar en consideración la voz de los agentes sociales que son parte de este proceso social, el trabajo de campo consistió en sistematizar la experiencia en el contexto educativo de 16 jóvenes que han meditado, 10 profesores y profesoras que han utilizado la meditación en sus clases, y 10 voluntarias y voluntarios de Manos sin Fronteras que participaron en las actividades de meditación en las instituciones. Gracias a su colaboración activa en la experiencia, se recogieron sus opiniones y percepciones, las cuales son indispensables para el análisis. Sus aportes fueron sistematizados a través del análisis sociológico del sistema de discursos (Callejo Gallego, 2010). El análisis de datos se realiza también en base a la teoría fundamentada; es decir que las respuestas van generando la teoría, si bien esta se contrasta con las fuentes consultadas previamente. Los instrumentos contaron con preguntas cerradas y abiertas, y fueron diseñados en base a la relevancia apuntada en la bibliografía, pero también gracias a lineamientos de entrevistas previas a maestras y maestros que utilizan la técnica de la meditación en el aula.

Si bien se trata de un estudio inminentemente cualitativo, se cuantifican las respuestas en porcentajes con el fin de apreciar la percepción del conjunto. Es decir que prima lo cualitativo, pero al haber preguntas cerradas esto da lugar a una cuantificación (los porcentajes citados en el texto aplican sobre el total de participantes); si bien esta no tiene valor estadístico, es un indicador interesante para futuros estudios. Estos datos dan fe de la validez y credibilidad de los resultados; no obstante, se recomiendan futuros estudios para afinar las conclusiones.

A pesar de entrevistar a tres grupos (profesorado, estudiantado, voluntariado), las respuestas tienden a homogenizarse. Se han sistematizado de forma conjunta a la hora de cuantificar, en vista de no presentar diferencias significativas porgrupos. Cuando resulta relevante se especifica; de otra forma las respuestas son referidas sencillamente como "participante" y el número que consta en los registros, permitiendo con esto también respetar los términos de privacidad y anonimato acordados en los protocolos de ética.

Las instituciones a las que pertenecen las experiencias referidas son las siguientes:

- Brasil: Nucleo de Educação Infantil Carinhoso NICC, Casa do Menino (instituição filantrópica, Organização da Sociedade Civil, OSC), Escola Municipal Eny Caldeira, Escola Municipal CEl Francisco Klemtz, Escola Municipal Eny Caldeira, Escola Municipal Coronel Durival Britto e Silva, Escola Estadual de Período Integral Major Arcy, Escolas do Brasil, Colégio Águia de Prata - unidade I.

- Colombia: Escuela de la República de Colombia, Escuela Secundaria Gabriela Mistral, Institución Educativa Nuestra Señora de Chiquinquirá Roldanillo, Colegio Empresarial de Los Andes Neiva, Liceo Benalcázar de Cali, Universidad Nacional de Medellín, Universidad Uniminuto de Bogotá. 
http://doi.org/10.15359/ree.25-3.24

http://www.una.ac.cr/educare

educare@una.ac.cr

- México: Universidad de Guadalajara, Centro Universitario de Ciencias de la Salud CUCS, CUCEl, Ciencias de Movimiento Humano, UDG, Universidad de Guadalajara-CUAAD Artes Plásticas.

- Guatemala: Escuela Oficial Urbana de Niñas República de Colombia, Universidad San Carlos de Guatemala (USAC).

- Honduras: Instituto Nimia Baquedano; Universidad Nacional Autónoma de Honduras (UNAH).

- Italia: Instituto Parini.

En todos estos centros se identifican los siguientes factores de riesgo según la percepción de los actores: fracaso escolar, amigos en situación de riesgo de llevar a cabo conductas antisociales o autodestructivas, aislamiento social, y dificultades para las relaciones sociales. Por otro lado, entre los factores protectores que destacaron en la investigación están las buenas experiencias escolares y las relaciones con iguales, la implicación en el juego, y los buenos resultados en los estudios.

Por lo tanto, es importante comenzar por indicar que los agentes y las agentes consideran que en su centro educativo hay estudiantes con dificultades para las relaciones sociales (75\%), en situación de fracaso escolar (50\%), con conductas antisociales o autodestructivas $(44,4 \%)$ y aislados socialmente (38,9\%). Es decir que, según la percepción de los sujetos involucrados en este estudio, factores considerados de riesgo se encuentran presentes de forma significativa en las instituciones educativas que frecuentan. Sin embargo, estos rasgos -tal como se verá más adelante en detalle- se ven atenuados una vez realizadas las prácticas de meditación, en especial al realizarlas de forma constante, lo que impacta tanto en el alumnado como en el profesorado. Este testimonio de una voluntaria es en tal sentido indicativo: "En esta localidad los jóvenes tienen conductas separatistas y autodestructivas. Pero al mes y medio, los jóvenes colaboraban. Se notó que los profesores escuchaban más y los alumnos se dejaban guiar si el caso lo requería" (Participante 26).

Las meditaciones descritas en el trabajo de campo comprenden lapsos de tiempo variables y discurren entre el 2016 y el 2019. Las edades oscilan desde los 3 años en preescolar, hasta estudiantado universitario mayor de 18. Fueron realizadas ya sea todos los días, meditando 5 minutos antes de empezar cada curso, o como experiencias puntuales. Las actividades de meditación tuvieron lugar en grupos que iban desde 14 estudiantes hasta 7000 participantes. Las personas entrevistadas relatan que incluso cuando los grupos fueron numerosos hubo buena participación y disposición para que cada cual realizara plenamente la meditación.

No obstante, se considera en estudios previos que lo ideal es que el ejercicio de meditación se practique todos los días (Almeida y Stadnik, 2017). Justamente, se ha planteado que la violencia escolar baja el desempeño académico del curso completo (Román y Murillo, 
http://doi.org/10.15359/ree.25-3.24

2011), por lo que las acciones con esfuerzos continuados resultan sumamente relevantes. La investigación más reciente refuerza la importancia de establecer estrategias de apoyo a nivel del curso completo, para promover una mejor relación dentro de los grupos.

Justamente, los estudios de Goldman y Davidson (2017) demuestran resultados similares en el sentido de cambios que van más allá de la sensación placentera que brinda la meditación. Afirman que se logran cambios positivos en los rasgos de la personalidad que pueden permanecer durante años. Recomiendan, a la par de una práctica asidua, una guía y retroalimentación para poder entrenar la mente y lograr cambios positivos. En este sentido, los espacios educativos se revelan como sitios privilegiados para tal actividad.

\section{Hallazgos}

\section{Mejor ambiente escolar}

Un profesor del Instituto Parini (Podenzano, provincia de Piacenza, Italia), al relatar su experiencia con estudiantes de 11-12 años, afirma que "la meditación ha mejorado el clima escolar: están más tranquilitos en los momentos que requieren más atención, hacen menos ruido y se escuchan más entre ellos y al profesor" (Participante 11). Este no es un hecho aislado, pues los estudios previos de monitoreo de experiencias similares de meditación en contextos educativos han recibido muy buena acogida, tanto por el personal educativo como por parte del estudiantado. Así lo refieren los testimonios compilados por otras investigaciones, especificando que la pedagoga y la dirección de la institución elogiaron mucho al proyecto, compartiendo los alcances obtenidos en la niñez y la alegría de la institución para acogerlo (Almeida y Stadnik, 2017).

En el presente trabajo, casi un $70 \%$ de las personas participantes considera que la meditación en el centro educativo ha propiciado buenas experiencias escolares. Según el criterio de uno de los participantes, "la meditación ha tenido efectos inmensamente positivos en el ámbito escolar: niños y niñas están en calma y tranquilidad, con lo cual tienen mejores resultados en el aprendizaje y las interacciones sociales" (Participante 11).

Efectivamente, la opinión generalizada es que con la meditación el ambiente educativo mejora, logrando "más tranquilidad y armonía entre los niños y las niñas" (Participante 10). Concretamente, casi un $60 \%$ afirma que la meditación predispone a una mayor colaboración y apoyo entre el grupo: "Ha integrado a todo el grupo y ha permitido que se desarrollen diferentes habilidades" (Participante 27).

En porcentajes similares se respondió que permite más y mejores relaciones con iguales (61,1\%). Al "mejorar las relaciones con los compañeros" (Participante 30), "baja el estrés, la agresividad, centrándolos en las actividades que se realizan. Siendo solidarios a la hora de materiales. Reconocen que abrazary dar amor es más importante que pelear" (Participante 34). 
http://doi.org/10.15359/ree.25-3.24

http://www.una.ac.cr/educare

educare@una.ac.cr

Asimismo, el estudiantado hace eco de las mismas impresiones: "ayuda a relajarte en momentos de estrés y a pensar mejor las cosas antes de hacerlas; me siento mejor" (Participante 25). El estado de bienestar y relajación se asocia a la posibilidad de prestar atención a lo que ocurre en clase; es decir, a mejorar los niveles de concentración en las actividades educativas ("Menor estrés y más atención", Participante 3; "poner más atención a la clase”, Participante 22; "Me relaja y me hace poner mayor atención", Participante 17).

Existe una relación probada a través de varios estudios que muestran que la intimidación y la violencia entre pares tiene un vínculo con ciertos factores de determinan los resultados escolares (Román y Murillo, 2011). Es decir que al mejorar las relaciones entre pares debería mejorar el rendimiento académico. Ese parece ser justamente el resultado según el decir del profesorado: "los estudiantes están más dispuestos a aceptar a sus compañeros y se percibe más armonía en el grupo para las actividades académicas" (Participante 20).

En vista de que la violencia es una de las grandes problemáticas que envuelve a los centros escolares a nivel mundial, y se ve directamente reflejada en el rendimiento académico (Kibriya et al., 2011), los esfuerzos que coadyuven a su disminución y prevención deben ser puestos a la luz entre las mejores prácticas. Por eso cabe resaltar que la meditación en los centros educativos "Baja los niveles de agresividad, mejora la comunicación, la concentración, mejora el estado de ánimo y la autoestima. Mejoran los que sufren de ansiedad y dolores de cabeza o insomnio" (Participante 15).

\section{Mejor desempeño académico}

En lo que concierne el ámbito educativo, varios estudios demuestran que tanto agresores o agresoras como víctimas tienen un menor rendimiento académico (Morcillo Morcillo, 2012). Por lo tanto, vale la pena recalcar los beneficios de la meditación que se encuentran relacionados, según lo perciben las personas participantes, con elementos claves para fomentar un entorno que propicie un clima adecuado para el proceso de aprendizaje y enseñanza. Una profesora de la Escola Estadual de Período Integral Major Arcy (São Paulo, Brasil), relatando su experiencia con estudiantes entre 11 y 18 años, afirma que el alumnado "mejora sus niveles de concentración, memoria, atención, paciencia, sensibilidad, intuición, sueño, salud, equilibrio emocional, y relaciones inter e intra personales" (Participante 27).

Justamente, más del $61 \%$ sostiene que la práctica de la meditación en el ámbito escolar mejora el desempeño académico. Se considera incluso "una herramienta pedagógica eficiente" (Participante 8). De hecho, más de la mitad de las personas participantes arguye que permite mejores resultados en el aprendizaje (52,8\%) y que amplía las posibilidades de aprender (50\%): "Se relajan mejor, por ejemplo, antes de un examen, antes de hacer una actividad de aula" (Participante 36). La meditación, por lo tanto, prepara al grupo para las actividades académicas. 
http://doi.org/10.15359/ree.25-3.24

Esto coincide con las conclusiones del biólogo molecular Ricard (2009), quien afirma que:

La meditación es una práctica que permite cultivar y desarrollar ciertas cualidades humanas fundamentales, de la misma manera que otras formas de entrenamiento nos enseñan a leer, a tocar un instrumento de música o a adquirir cualquier otra aptitud (p. 12).

La mitad de las personas que colaboraron en este estudio expresó que mejora la calidad de las clases, tal como lo refleja este testimonio: "Nos permite relajar nuestra mente y cuerpo para dejar en segundo plano algunas preocupaciones externas a la clase y concentrarse en lo que se hará en esa materia" (Participante 16). Utilizándola al inicio de la jornada puede tener aspectos positivos que repercutan en la motivación, tal como afirma una docente: "La meditación de 5 minutos tuvo gran acogida con mis estudiantes; la hacemos antes de iniciar las clases y noto cómo los chicos están más dispuestos. También aplico estimulación neural para ayudarles cuando presentan molestias de salud en clase" (Participante 29).

La estimulación neural (Instituto Internacional de Estimulación Neural yTerapias Naturales, 2020) es una técnica promovida igualmente por Manos sin Fronteras para utilizarse en el entorno educativo formal, entre otros. La organización la describe de esta manera:

La estimulación neural es una técnica rápida y efectiva: con solo 5 minutos diarios la persona podrá experimentar los beneficios a su bienestar. Es una técnica natural de aplicación universal que actúa sobre los 3 pilares principales del cuerpo: sistema inmunológico, nervioso y sanguíneo (Manos Sin Fronteras, 2020, párr. 1-2).

\section{Cultura de paz}

La violencia en el ámbito educativo tiene repercusiones en términos académicos, pero sabemos también que la calidad de la enseñanza depende en gran medida del personal técnico y pedagógico. El ambiente escolar se ve afectado por la calidad de las relaciones interpersonales a todos los niveles (Abramovay, 2005). Sin embargo, con la meditación, "hay mayor armonía entre alumnos y maestros" (Participante 6). La meditación "mejora el vínculo del profesor con la clase y ejercita el respeto entre el alumnado, además de aumentar la sensación de paz del estudiantado" (Participante 12), aspecto en el que coinciden varias personas.

Una profesora de la Escola Municipal Eny Caldeira (Curitiba, Brasil), relatando su experiencia con estudiantes de 6 a 10 años, sostiene que niños y niñas "sienten la motivación de meditar porque ven sus beneficios, tales como tranquilidad, relajamiento y paz" (Participante 13); e incluso enfatiza en que hay quien relata que hace la meditación con la aplicación en casa.

En el entorno educativo formal existe la necesidad de crear mecanismos de negociación sobre los reglamentos y las normas internas, así como concientizar al profesorado sobre las 
http://doi.org/10.15359/ree.25-3.24

http://www.una.ac.cr/educare

educare@una.ac.cr

diversas maneras para lograrlo. Un tercio considera que con la meditación se obtiene mayor respeto de las normas, al lograr "una mejor convivencia en general y relajación" (Participante 23).

El aprendizaje está también influido por la parte afectiva y emocional, por lo que es indispensable escuchar y conversar. Justamente un docente comenta que: "La meditación hizo que hubiese menos impulsividad en las relaciones interpersonales, menos ansiedad. Los adolescentes mejoraron su capacidad de escucha. La hicimos todos los días antes de comenzar las actividades. Los adolescentes respondieron positivamente al propósito de la meditación" (Participante 8).

Efectivamente, casi el $90 \%$ refiere que la meditación permite una mejor calidad de escucha y comunicación (88,9\%). Resultados similares fueron obtenidos en investigaciones previas sobre experiencias de meditación en el contexto educativo formal. En los primeros meses del proyecto se percibió que, a pesar de que niñas y niños no tenían ningún tipo de contacto con la meditación anteriormente, se acostumbraron de forma satisfactoria a la actividad. De tal manera, podemos decir que la niñez la integra sus vivencias y los varios saberes de forma expresiva a través de la meditación. Por medio de grupos de discusión, realizados antes y después de practicar la meditación, niñas y niños tuvieron la oportunidad de exponer sus pensamientos y emociones, aprendiendo así a dirigirse respetuosamente entre ellos, a compartir, colaborar, turnarse e inhibir comportamientos agresivos e intrusivos, esperando su momento para hablar y experimentar ser escuchados por todos los presentes (Piccinin, et al., 2018).

La participación de alumnos y alumnas en la creación de un ambiente de confianza y comunicación es también clave, ya que el clima de convivencia en las aulas es un factor relacionado con el rendimiento del estudiantado: "La meditación ha mejorado el clima escolar. Los alumnos se escuchan más entre ellos y al profesor. Hacen menos ruido, están más tranquilitos en los momentos que requieren más atención" (Participante 18).

Es decir que se abona para lograr una convivencia pacífica $(61,1 \%)$ : "Hace mejores a las personas, menos conflictivas" (Participante 31); "más agradables y sociables" (Participante 4). La convivencia es uno de los núcleos fundamentales de nuestra vida y de nuestro desarrollo. Las personas que colaboraron en este estudio afirman que "el ambiente se ha vuelto más tranquilo y positivo" (Participante 19), y que la experiencia proporciona "bienestar y tranquilidad" (Participante 5).

No basta con reaccionar frente a la violencia, sino que es necesario construir una convivencia positiva. Repercutiendo "en la convivencia y comprensión hacia los demás" (Participante 32), más de la mitad de las personas entrevistadas -en un porcentaje similar al anterior- afirma que fomenta la cultura de paz (58,3 \%), "logrando un ambiente más pacífico" (Participante 24). 


\section{Resiliencia}

Una voluntaria de la Escuela Oficial Urbana de Niñas República de Colombia (Guatemala, Guatemala), relatando su experiencia con estudiantes de 6 a 14 años en escuela de vacaciones, comenta que con la meditación las alumnas "bajaron sus niveles de estrés y agresividad, logrando centrarse en las actividades y mostrar solidaridad a la hora de compartir materiales" (Participante 34). Casi la mitad de las personas participantes considera que la meditación promovida por Manos sin Fronteras ayuda a superar las contrariedades (44,4\%). "En Honduras tenemos una ola de suicidios; creo que la meditación es una gran herramienta para contrarrestar este mal. En lo personal me ayuda a mantenerme en paz aun cuando mi entorno está en conflicto. Quiero que los demás conozcan esta herramienta" (Participante 9). Un tercio piensa justamente que permite percibir las dificultades como retos (33,3\%). Estos aspectos pueden jugar un rol decisivo para prevenir la violencia y fomentar la resiliencia.

Hay que brindar especial atención al entorno inmediato de la institución y a su ambiente interno, pues existe una interacción entre la escuela, la familia y la comunidad, además de ser pilares fundamentales de apoyo. La familia debe ser fuente de sensibilidad, disciplina y comunicación. En este sentido, es importante subrayar que "muchos de los estudiantes compartieron la meditación con sus padres para que ellos también la hicieran" (Participante 20). Un tercio de las personas entrevistadas sostiene que la meditación ha promovido una mejor interacción entre el centro educativo y la familia. Una de las personas colaboradoras con el estudio comenta en su testimonio que "niños y niñas tienen la motivación de meditar porque sienten los beneficios, como tranquilidad, relajamiento y paz; algunos niños relatan que hacen la meditación con la aplicación del celular en la casa" (Participante 13). Justamente, la evidencia existente sugiere que los programas de prevención de violencia más rentables son aquellos que se realizan a través de las escuelas y los programas de desarrollo infantil temprano, conjuntamente con la capacitación de madres, padres y tutores. Lograr visibilizar a personas adultas significativas es también un factor de resiliencia, y casi un $20 \%$ considera que esta actividad lo permite.

Más de un tercio de las personas participantes $(36,1 \%)$ opina que el profesorado tiene mejor actitud gracias a las actividades de meditación: "nos ayuda con la clase a estar más relajados" (Participante 7). De hecho, una persona opina que le permite "tener más paciencia hacia los jóvenes" (Participante 35). El profesorado juega un papel clave a la hora de fomentar la resiliencia, la cual implica una buena disposición y pensamientos en positivo. Se trata de transformar los problemas y ayudar a solventarlos, viendo el conflicto como una oportunidad de enfrentar y transformar, entendiendo que los desafíos se encuentran en el camino para alcanzar objetivos comunes. Una catedrática opina:"Sinceramente, me da tranquilidad, y es buena para manejar el estrés, y más en esta etapa de la universidad que es cuando más problemas nos causa. Sin embargo, las meditaciones ayudan al alumnado a llevar una mejor calidad de estudio y tranquilidad" (Participante 14). 
http://doi.org/10.15359/ree.25-3.24

http://www.una.ac.cr/educare

educare@una.ac.cr

Para sintetizar los beneficios de la práctica, vale citar a la fundadora de Manos sin Fronteras:

Muchas veces cuando nos caemos o nos duele, puede ser el estómago, la espalda, el pie; pero si empezamos a respirar, poco a poco vamos tomando esa forma de meditar, el dolor se va pasando.

También mejora mucho la memoria porque nos hace cambiar de pensamiento. Automáticamente nuestra memoria se pone en funcionamiento; y es que meditar ralentiza el envejecimiento de nuestro cerebro, le ayuda a mantenerse joven, lo ayuda sobre todo a trabajar, a estar siempre activo, a calmar nuestra ansiedad, el estrés, nuestros pensamientos (La Jardinera, 2020, “¿Quién soy yo”, párr. 5-7).

\section{Conclusión}

No existe un solo factor que explique por qué una persona se comporta de manera violenta y otra no, ni por qué un centro educativo o una comunidad se encuentran inmersos en la violencia mientras otros viven en paz. De hecho, la complejidad de la violencia remite a factores biológicos, sociales, culturales, económicos y políticos (OMS, 2002). Sin embargo, el impacto a largo plazo de exponerse a la violencia tiene un precio y, por tanto, merece el esfuerzo reflexionar e investigar al respecto. Haberla vivido en la infancia está relacionado con su uso y aceptación; de allí la importancia de intervenir a tiempo, brindando herramientas eficaces para encontrar respuestas creativas que permitan otro tipo de vivencias.

La convivencia es uno de los núcleos fundamentales de la vida y desarrollo humano. Un ambiente educativo hostil perjudica las relaciones entre las personas, afecta el ejercicio profesional del equipo técnico-pedagógico y el rendimiento académico. La meditación implementada por Manos sin Fronteras, en diferentes contextos educativos a nivel mundial, ha permitido resaltar una serie de factores que se pueden considerar protectores ante la violencia, al mostrarse capaz de favorecer el desarrollo individual y grupal, reducir los efectos de circunstancias desfavorables e incluso revertir circunstancias potencialmente negativas y transformarlas.

Según la percepción del estudiantado, profesorado y voluntariado que ha colaborado en este estudio, la meditación desarrolla aspectos decisivos para prevenir la violencia y fomentar la resiliencia. Incluso factores considerados de riesgo parecen atenuarse a través de sencillas prácticas de meditación, cuando estas se realizan de forma constante. A continuación, el resumen de tales percepciones.

La meditación fomenta paciencia, sensibilidad, equilibrio emocional, bienestar y relajación. Al haber calma y armonía, bajan los niveles de estrés, agresividad, ansiedad e impulsividad. Hace mejores a las personas, que se tornan menos conflictivas, más agradables, comprensivas, respetuosas y sociables, promoviendo la convivencia pacífica. Mejora el estado de ánimo 
http://doi.org/10.15359/ree.25-3.24

y la autoestima. Esto permite una mejor interacción social: más y mejores relaciones entre pares, aceptación, respeto, colaboración, apoyo, solidaridad e integración. Un gran número de estudiantes compartió la meditación con sus familiares, mejorando la interacción entre el centro educativo y su entorno.

Al haber menos ruido y más tranquilidad, hay una alta calidad de escucha y comunicación creando un ambiente de confianza, verdaderas bases de la cultura de paz. Al propiciar la comunicación, concentración, atención, memoria, intuición y motivación puede considerarse la meditación como una herramienta pedagógica que amplía las posibilidades de aprender, permitiendo mejores resultados, calidad de las clases y del estudio.

A nivel del profesorado, fomenta la buena actitud, paciencia y disposición positiva, características que visibilizan a personas adultas significativas. Esto se considera como un factor de resiliencia, fundamental para facilitar y ayudar a superar contrariedades, así como revertir circunstancias potencialmente negativas y transformarlas. De allí quizá que se obtenga un mayor respeto de las normas.

Las razones expuestas son los suficientemente relevantes para valorar la meditación en entornos educativos como un factor protector y de resiliencia, por fomentar las capacidades antes descritas, las cuales se constituyen en verdaderos escudos protectores ante la adversidad, atenuando efectos negativos e incluso transformando y promoviendo la superación de la situación difícil, que potencialmente puede propiciar violencia. Los resultados obtenidos alientan no solo a realizar más estudios sobre meditación y estimulación neural, sino incluso a ir más allá de los contextos educativos con el fin de determinar su alcance, en tanto respuesta eficaz contra la violencia, con buenas probabilidades de lograr un cambio significativo en entornos que presentan factores de riesgo.

Los centros educativos son grandes aliados para desarrollar resiliencia y prevención, por ser la interfaz local entre la ciudadanía y el Estado para canalizar herramientas. Desde los sistemas educativos es posible transmitir, mediante una estrategia sistemática, las destrezas sociales necesarias para formar a las ciudadanas y los ciudadanos. De otra manera, en lugar de ser el lugar en el que se gesta el cambio necesario, varios expertos en la temática señalan que las escuelas pueden constituir un espacio de reproducción de las desigualdades sociales y la violencia.

\section{Declaración de Material complementario}

Este artículo tiene disponible, como material complementario:

-La versión preprint del artículo en https://doi.org/10.5281/zenodo.4414879 
http://doi.org/10.15359/ree.25-3.24

http://www.una.ac.cr/educare

educare@una.ac.cr

\section{Referencias}

Abramovay, M. (2005) Victimización en las escuelas. Ambiente escolar, robos y agresiones físicas. Revista Mexicana de Investigación Educativa, 10(26), 833-864. http://www.scielo.org.mx/ scielo.php?script=sci abstract\&pid=S1405-66662005000300833\&lng=es\&nrm=iso

Abt, T. y Winship, C. (2016). What works in reducing community violence: A meta-review and field study for the northern triangle. United States Agency for International Development.

Almeida, T. P. y Stadnik, A. M. W. (2017). Práticas de meditação na infância. Actas del 7o Seminário de Extensão e Inovação da UTFPR (pp. 1-8). Universidade Tecnológica Federal do Paraná.

Ayestas, M. N. (2007). Prevención de violencia en centros educativos: Módulo I: Violencia y criminalidad. PNUD. https://tzibalnaah.unah.edu.hn/handle/123456789/254

Banco Mundial (2011). Crimen y violencia en Centroamérica: Un desafío para el desarrollo (Vol. 1). Autor. https://openknowledge.worldbank.org/handle/10986/2744?locale-attribute=es

Benbenishty, R. y Astor, R. A. (2005). School violence in context: Culture, neighborhood, family, school, and gender. Oxford University Press.

Bronfenbrenner, U. (1979). The ecology of human Development: Experiments by nature and design. Harvard University Press. https://khoerulanwarbk.files.wordpress.com/2015/08/urie bronfenbrenner the ecology of human developbokos-z1.pdf

Callejo Gallego, J. (2010). Fernando Conde Gutiérrez del Álamo, Análisis sociológico del sistema de discursos. CIS, Madrid, 2009. Empiria, Revista de Metodología de Ciencias Sociales, (20), 246-251. https://doi.org/10.5944/empiria.20.2010.2049

Consejo Noruego para Refugiados (2016). ¿Esconderse o huir? La situación humanitaria y la educación en Honduras. Convención sobre los derechos del niño. Autor. https://reliefweb. int/report/honduras/esconderse-o-huir-la-situaci-n-humanitaria-y-la-educaci-n-en$\underline{\text { honduras }}$

Eumedito (2020). 5 minutos, yo medito. http://www.eumedito.org/es/

Ferreira, R. C. (2016). A meditação como forma de promover as relações de amizade entre as crianças [Tesis de maestría]. Universidade do Algarve. https://www.google.co.cr/url?sa= $t \& r c t=j \& q=\& e s r c=s \&$ source $=$ web\& $c d=\& c a d=r j a \& u a c t=8 \& v e d=2 a h U K E w i Y 5 o n G j K n x A h W$ dRDABHQcsCsgQFjAAegQIBhAF\&url=https\%3A\%2F\%2Fsapientia.ualg.pt\%2Fbitstream \%2F10400.1\%2F8666\%2F1\%2FA\%2520medita\%25C3\%25A7\%25C3\%25A30\%2520como \%2520forma\%2520de\%2520promover\%2520a\%2520rela\%25C3\%25A7\%25C3\%25B5es \%2520de\%2520amizade\%2520entre\%2520as\%2520crian\%25C3\%25A7as\%2520\%2520\%2520V\%2520\%2520final.pdf\&usg=AOvVaw2sQInJck1tZf7nQZmR9ZRh 
http://doi.org/10.15359/ree.25-3.24

Gaxiola Romero, J. y Frías Armenta, M. (2008). Un modelo ecológico de factores protectores del abuso infantil: Un estudio con madres mexicanas. Medio Ambiente y Comportamiento Humano, 9(1-2), 13-31. https://dialnet.unirioja.es/servlet/articulo?codigo=2789614

Goldman, D. y Davidson, R. J. (2017). Altered traits: Science reveals how meditation changes your mind, brain, and body. Penguin Publishing.

Instituto Internacional de Estimulación Neural y Terapias Naturales (2020). Tratamientos exclusivos. http://institutoestimulacion.com/tratamientos-exclusivos/

Kibriya, S., Tkach, B., Ahn, J., Valdez González, N., Xu, Z. y Zhang, Y. (2011). The effects of schoolrelated gender-based violence on academic performance. Evidence from Botswana, Ghana \& South Africa. USAID.

La Jardinera. (17 de enero, 2020). “Camino recto, camino de Luz". |https://www.facebook.com/ notes/la-jardinera/camino-recto-camino-de-luz/2211026175641083/

Manos Sin Fronteras. (2020). Estimulación neural. https://www.msfint.com/es/estimulacionneural/

Morcillo Morcillo, B. (2012) La violencia escolar y su influencia sobre el rendimiento académico [Tesis de maestría]. Universidad Internacional de la Rioja. https://reunir.unir.net/ handle/123456789/628

Nichel, M., Stadnik, A. M. W., Garanhani, M. C. y Ulbricht, L. (2013). Aspectos qualitativos das ações do projeto Meditando na infância. Actas del 8 Seminário de Iniciação Científica e Tecnológica da UTFPR. Universidade Tecnológica Federal do Paraná.

Nichel, M. y Stadnik, A. M. W. (2014). O exercício de meditação na infância: A trajetória e as contribuições de um projeto de extensão. Trabajo presentado en $4^{\circ}$ Seminário de Extensão e Inovação da UTFPR. Universidade Tecnológica Federal do Paraná.

Nichel, M. y Stadnik, A. M. W. (2016). Projeto meditando na infância: A capacitação do corpo docente como proposta de extensão e formação continuada. $6^{\circ}$. Seminário de Extensão e Inovação da UTFPR. Universidade Tecnológica Federal do Paraná.

Organización Mundial de la Salud. (2002). Informe mundial sobre la violencia y la salud. Autor.

Organización Mundial de la Salud. (2017). Violencia. Autor.

Organización Mundial de la Salud. (8 de junio, 2020). Violencia juvenil. http://www.who.int/ mediacentre/factsheets/fs356/es/ 
http://doi.org/10.15359/ree.25-3.24

http://www.una.ac.cr/educare

educare@una.ac.cr

Organización Panamericana de la Salud. (2016). Informe sobre la situación mundial de la prevención de la violencia 2014. Autor. https://iris.paho.org/handle/10665.2/28233?localeattribute $=\mathrm{es}$

Piccinin, C. F., de Almeida, T. P. y Stadnik, A. M. (2018). O cata-vento como coadjuvante na prática de meditação com crianças pré-escolares. $8^{\circ}$ Seminário "EU MEDITO na educação". Universidade Tecnológica Federal do Paraná. Universidade Tecnológica Federal do Paraná.

Ricard, M. (2009). El arte de la meditación. Urano.

Rocha, M. D. (2014). Meditando e brincando: Práticas de meditação na educação infantil [Tesis de licenciatura]. Universidad Federal do Río Grande do Sul. https://www.lume.ufrgs.br/ handle/10183/117538

Román, M. y Murillo, F. J. (2011). América Latina: Violencia entre estudiantes y desempeño escolar. Revista Cepal, (104), 37-54. https://repositorio.cepal.org/bitstream/ handle/11362/11458/104037054 es.pdf?sequence=1\&isAllowed=y 